\title{
Comment on the magnetic dipole hyperfine interaction in the gold atom ground state
}

\author{
Jacek Bieroń $^{1,2,4}$, Charlotte Froese Fischer ${ }^{2}$, Per Jönsson ${ }^{3}$ \\ and Pekka Pyykkö ${ }^{4}$ \\ ${ }^{1}$ Instytut Fizyki imienia Mariana Smoluchowskiego, Uniwersytet Jagielloński, Reymonta 4, \\ 30-059 Kraków, Poland \\ ${ }^{2}$ National Institute of Standards and Technology, Gaithersburg, MD 20899-8420, USA \\ ${ }^{3}$ Nature, Environment, Society, Malmö University, S-205 06 Malmö, Sweden \\ ${ }^{4}$ Department of Chemistry, University of Helsinki, POB 55 (A I Virtasen aukio 1), 00014 Helsinki, \\ Finland \\ E-mail: Bieron@uj.edu.pl
}

Received 16 April 2008, in final form 5 May 2008

Published 28 May 2008

Online at stacks.iop.org/JPhysB/41/115002

\begin{abstract}
The multiconfiguration Dirac-Hartree-Fock (MCDHF) model has been employed to calculate the magnetic dipole hyperfine constant $A$ of the $5 \mathrm{~d}^{10} 6 \mathrm{~s}^{2} \mathrm{~S}_{1 / 2}$ ground state of atomic gold. Electron correlation effects contribute more than $20 \%$ to the total value of $A$. We investigated the effects of single, double, and a subset of triple substitutions. The calculations reveal strong cancellations between one-, two- and three-particle correlation effects. It is demonstrated that in the case of the ground state of atomic gold the three-particle effects are comparable in size to the one- and two-particle ones.
\end{abstract}

\section{Introduction}

The Dirac-Coulomb-Breit Hamiltonian, in principle, provides an excellent description of the atomic and molecular structure. Difficulties arise in applying it to systems with many electrons, if electron correlation effects are important. The magnetic dipole hyperfine coupling constants of neutral or nearly neutral atoms are particularly challenging. A simple qualitative analysis shows that the largest magnitude of orbitals for unpaired electrons is in the valence region, whereas most of the actual hyperfine coupling arises in the K-shell region. The influence of the nuclear charge distribution and the magnetic distribution inside the nucleus is also important for isotopes of heavy elements. Thus in various ways we are gauging the entire electronic and nuclear system.

The gold atom is a case in point. The relativistic effects (defined as the differences between using the Dirac and the Schrödinger one-electron equation) which explain the chemical differences between gold and silver, have a local 'gold maximum' [1] for Group-11 elements. The maximum arises from the interacting relativistic and shellstructure effects [2]. The core-valence electron correlation is known to be especially important. Good experimental values for the magnetic dipole hyperfine constant $A$ are available. The ground-state $A$ is 3049.660092 (7) $\mathrm{MHz}$ [3]. A further aspect is the intense current activity on the theoretical chemistry of gold [4-6]. Getting A 'right for the right reason' is a useful test of current computational methods.

A related challenge is the electric quadrupole hyperfine coupling constant, $B$, which can potentially yield improved values of the nuclear quadrupole moment, $Q$. The current standard value $0.547(16)$ barn $\left(1\right.$ barn $\left.=10^{-28} \mathrm{~m}^{2}\right)$ for $Q\left({ }^{197} \mathrm{Au}\right)$ comes from muonic states, but new 'atomic' or 'molecular' values are being proposed. Perhaps the most reliable new value is the 'atomic' one, $Q\left({ }^{197} \mathrm{Au}\right)=0.521(7)$ barn [7].

Against this background it is interesting that Song et al [8] reported a calculation of $A$ and $B$ hyperfine values for the $6 \mathrm{~s}$ and $6 \mathrm{p}$ states of $\mathrm{Au}$, agreeing with the experiment at the per cent level. In the present paper we consider the $A$ constant alone, but go to much larger active spaces than those used by Song et al.

In first-order perturbation theory, only single substitutions from the Dirac-Hartree-Fock (DHF) reference state contribute 
to the hyperfine energy operator $[9,10]$, so they usually comprise the dominant part of the hyperfine energy correction. On the other hand, double substitutions dominate energetically over valence correlation corrections and, consequently, the virtual orbitals obtained in fully correlated variational calculations are optimized predominantly through the effects of double substitutions on the total energy. The approach which is often utilized is based on single and restricted double substitutions. The restriction constrains double substitutions in such a way that at least one electron from the pair must originate from valence shell(s). In the case of gold this means that at most one electron is substituted from $1 \mathrm{~s} 2 \mathrm{sp} 3 \mathrm{spd} 4 \mathrm{spdf} 5 \mathrm{spd}$ shells, the remaining one from the $6 \mathrm{~s}$ shell. In our previous papers on hyperfine structure shifts and on nuclear electric quadrupole moments (see e.g. [11-14]) this 'single and restricted double substitutions' approach was often used, and at later stages the calculations were augmented by unrestricted double substitutions. The latter were usually applied in a configuration-interaction mode, i.e. without changing the radial factor of the one-electron spinorbitals.

The calculations are usually carried out in steps, where at each step the size of the multiconfiguration expansion is increased in a systematic manner. The convergence of the calculated expectation value(s) serves to control the scope of the calculations (see the above-quoted papers for details). In some cases this methodology permits the determination of hyperfine structure constants with an accuracy of about 1$3 \%$. The limiting factor is the ability to accurately model and control the two-, three- and higher many-body effects. The gold atom represents one of the more difficult cases for the following reasons. The atom is essentially an alkali-like system with a polarizable $5 \mathrm{~d}^{10}$ shell. The direct and indirect effects of relativity bring the outer $\mathrm{d}$ shell much closer, radially and energetically, to the valence s shell than in homologous silver and copper atoms [4, 15]. This in turn increases the polarization of the $5 \mathrm{~d}^{10}$ shell by the valence electron, relative to silver and copper. The interaction of several closed s shells with the valence s electron and, indirectly, among themselves, has also a sizeable effect on the magnetic hyperfine interaction [16]. Estimates of the polarization effects on the hyperfine structure from each of the closed shells can be obtained in the single substitution picture using non-orthogonal orbitals [17]. Although this approach gives a surprisingly good description in many cases, it is important to include higher order effects. This is complicated by the fact that different higher order effects, as well as the contributions from the different polarized shells, often cancel each other and an unbalanced calculation may lead to a completely erroneous value [18]. For nominally one-electron systems the core-valence electron correlation effects lead to the contraction of the valence orbital, which overestimates the hyperfine structure. The unrestricted double substitutions affect the hyperfine structure in two ways: directly through the configuration state functions (CSFs) themselves and also indirectly through the change of the expansion coefficients of the important configurations obtained by single excitations. By partitioning the configuration space into different subspaces it is possible to analyse these effects separately $[18,19]$. The overall effect of the double substitutions is to lower the calculated values of the hyperfine interaction constant. Three-particle effects are often very important. In a simple picture we can describe the wavefunction in terms of pair-correlation functions and the three-particle effects then account for the polarization of paircorrelation functions, leading to an increase of the hyperfine structure [20]. Four-particle effects are usually small and can often be neglected [18].

The purpose of the present paper is twofold. The $5 \mathrm{~d}^{10} 6 \mathrm{~s}$ ground state of gold is a doublet $\mathrm{S}$ system. The hyperfine interaction arises primarily from the spin interaction of an unpaired valence electron with the nuclear magnetic moment, but strong correlation effects are to be expected, since at the same time the valence electron polarizes the electronic core. The first objective of this paper is to study the role of one-, two- and three-particle correlation effects in the hyperfine interaction of the ground state. In the last few years several papers have appeared dealing with hyperfine interaction in gold atoms [21, 22], as well as gold compounds and gold alloys [4-6]. The methodology described above (multiconfiguration Dirac-Hartree-Fock with single and restricted double substitutions) was used to obtain the hyperfine expectation values of the ground and two excited states of the gold atom in two of the most recent theoretical papers $[8,23]$. The purpose of this paper is to check the validity of this approach. It can be considered a test of 'singlesubstitution' or 'restricted double substitutions' approach to computation of the hyperfine constants in heavy atoms, and in particular in the gold atom. The second objective is, for the present calculations, to serve as a test for the planned evaluation of hyperfine expectation values of the excited states of gold, in order to determine the nuclear quadrupole moment $Q\left({ }^{197} \mathrm{Au}\right)$.

\section{Theory}

We used a slightly modified version of the General Relativistic Atomic Structure Package (GRASP) [24] program for this work. The theory [25-27] and method of calculation $[28,29]$ have been described elsewhere. The numericalgrid-based wavefunction $\Psi(\Gamma P J)$ for the ground state $\Gamma\left(5 \mathrm{~d}^{10} 6 \mathrm{~s}^{2} \mathrm{~S}_{1 / 2}\right)$ of ${ }_{79}^{197} \mathrm{Au}$

$$
\Psi(\Gamma P J)=\sum_{k}^{N C F} c_{k} \Phi\left(\gamma_{k} P J\right)
$$

was obtained as the self-consistent solution of the DiracHartree-Fock equations [30] in systematically increased multiconfiguration bases (of size NCF) of symmetry-adapted configuration state functions $\Phi\left(\gamma_{k} P J\right)$. Each $\Phi\left(\gamma_{k} P J\right)$ is an eigenfunction of parity $P$ and of total angular momentum $J$, and it represents a configuration state function for configuration $\gamma_{k}$. Configuration mixing coefficients $c_{k}$ were obtained through diagonalization of the Dirac-Coulomb Hamiltonian

$$
H_{D C}=\sum_{i} c \boldsymbol{\alpha}_{i} \cdot \boldsymbol{p}_{i}+\left(\beta_{i}-1\right) c^{2}+V\left(r_{i}\right)+\sum_{i>j} 1 / r_{i j} .
$$


where $V(r)$ is the monopole part of the electron-nucleus interaction. Effects of the Breit interaction and the quantum electrodynamics (QED) corrections were neglected, since they were outside the objective of the present paper and they are small in comparison to the omitted correlation effects. The coefficients $c_{k}$, together with the one-electron orbital set, provide a numerical representation of the state $\Psi(\Gamma P J)$ for the further calculation of the atomic expectation value of the magnetic dipole hyperfine constant $A$. All calculations were done with the nucleus modelled as a spherical ball, where a two-parameter Fermi distribution [31] was employed to approximate the radial dependence of the nuclear charge density. The nuclear magnetic dipole moment $\mu=0.145746(9) \mu_{N}$ of ${ }_{79}^{197} \mathrm{Au}$ (nuclear spin $I=3 / 2$ ) has been taken from the tables of Raghavan [32].

\section{Method of calculation}

We employed the method described as a systematic expansion of configuration set [28, 29], in which configuration state functions of a particular parity and symmetry are generated by substitutions from reference configurations to an active set of orbitals. The active set is systematically increased until the convergence of the calculated value of $A$ is obtained.

\subsection{Virtual orbital set}

Eight layers of virtual orbitals were generated-three layers with spdfgh symmetries and five (from 4th up to 8th virtual layer) limited to spdfg symmetries. Double substitutions from valence and core orbitals were allowed, with the restriction that at least one electron is substituted from the valence $6 \mathrm{~s}$ subshell, while the remaining one may be substituted from core 3 spd4spdf5spd orbitals. In each subsequent layer all occupied orbitals were open for substitutions, with the exception of $1 \mathrm{~s} 2 \mathrm{sp}$ orbitals. It should be noted that the notion of a 'layer' is somewhat different when applied to occupied (also referred to as spectroscopic) orbitals, as opposed to virtual (also referred to as correlation) orbitals. A core 'layer', i.e. a subset (often referred to as a shell) of occupied orbitals possessing the same principal quantum number, constitutes a set of oneelectron spin-orbitals which are clustered in space, as well as with respect to the one-electron energy values. On the other hand, virtual orbitals with the same principal quantum number are not necessarily spatially clustered, because their one-electron energy values do not have physical meaning and may vary widely, depending on the correlation effects, which a particular virtual orbital describes. Therefore a 'virtual layer' usually means a subset of the virtual set, generated in one step of the procedure, as described above. Such a 'layer' is often composed of orbitals with different angular symmetries. The notation used in the tables of the present paper reflects the above considerations, in the sense that occupied orbitals are listed by their principal and angular quantum numbers (i.e. 5 spd means three occupied orbitals of $\mathrm{s}, \mathrm{p}$, and d symmetry with principal quantum number $n=5$ ), while virtual orbitals are listed by angular symmetry and quantity (i.e. 5 spd means fifteen virtual orbitals-five of each
Table 1. Calculated value of $A$ for the ${ }^{2} S_{1 / 2}$ state of $A u$ as a function of multiconfiguration expansion; DHF $=$ uncorrelated Dirac-Hartree-Fock; $n=$ maximal principal quantum number within a virtual set, optionally followed by a letter indicating highest angular symmetry of the last virtual layer within a given set; 'layers' $=$ number of virtual orbitals of a particular symmetry; 'NCF' = size of multiconfiguration expansion.

\begin{tabular}{llrl}
\hline & \multicolumn{2}{c}{ Experiment } & $3049.660092(7)$ \\
\cline { 2 - 3 }$n$ & Layers & NCF & $A(\mathrm{MHz})$ \\
\hline 6 & DHF & 1 & 2344.55 \\
7 & 1spdfgh & 609 & 2753.58 \\
8 & 2spdfgh & 2304 & 2893.28 \\
9 & 3spdfgh & 5089 & 3135.88 \\
$10 \mathrm{~g}$ & 4spdfg3h & 8320 & 3150.58 \\
$11 \mathrm{~g}$ & 5spdfg3h & 12369 & 3205.60 \\
$12 \mathrm{~g}$ & 6spdfg3h & 17236 & 3221.97 \\
$13 \mathrm{~g}$ & 7spdfg3h & 22921 & 3228.10 \\
$14 \mathrm{~g}$ & 8spdfg3h & 29424 & 3230.08 \\
\hline
\end{tabular}

of the s, p, and d symmetries). The notation should always be analysed in the proper context. As an example, the notation 8 spdfg $3 \mathrm{~h}$ in the second column of the last line of table 1 means a virtual set composed of: eight virtual orbitals of $s$ angular symmetry, eight $\mathrm{p}$ virtual orbitals, eight $\mathrm{d}$, eight $\mathrm{f}$, eight $\mathrm{g}$, and three h virtual orbitals. Since there are six occupied orbitals of s symmetry, the total number of s orbitals is 14 . Therefore the maximal principal quantum number within the largest orbital set is also 14, as indicated in the first column of the last line of table 1. The last five layers of virtual orbitals are composed of spdfg symmetries, therefore a letter $\mathrm{g}$ is appended to the maximal principal quantum number of virtual sets in the first column of the last five lines of the table 1 .

Table 1 presents the value of $A$ of the ${ }^{2} S_{1 / 2}$ state of $\mathrm{Au}$ as a function of the size of the virtual orbital set, with the multiconfiguration expansions described above. The uncorrelated Dirac-Hartree-Fock result is about 23\% smaller than the experimental value, while the fully converged-within this approximation - result overshoots the experimental value by $6 \%$.

\subsection{Substitutions from $1 \mathrm{~s} 2 \mathrm{sp}$ orbitals}

After generating the virtual orbital set, all orbitals were frozen and further calculations were carried out in the configurationinteraction (CI) scheme. First, the effects of $1 \mathrm{~s} 2 \mathrm{sp}$ orbitals were evaluated in separate CI calculations. They are presented in table 2, together with the contributions of all other occupied orbitals of the gold atom. The orbitals which were open for single and restricted double substitutions to the full virtual set are listed in the first column. The contributions of individual orbitals (i.e. of the leftmost orbital in the first column) are listed in the last column and are presented in a graphical form in figure 1 . The individual contributions of the $2 \mathrm{~s}$ and $1 \mathrm{~s}$ shells to the total $A$ value are about $0.52 \%$ and $0.14 \%$, respectively. A comparison with table 2 in the paper by Dougherty et al [33] shows good agreement with respect to the contribution of the $2 \mathrm{~s}$ shell, while the contribution of the $1 \mathrm{~s}$ shell paper is $0.22 \%$, somewhat larger than in our calculation. It should be noted that the combined contribution of $1 \mathrm{~s} 2 \mathrm{sp}$ shells is about $0.7 \%$ of the 


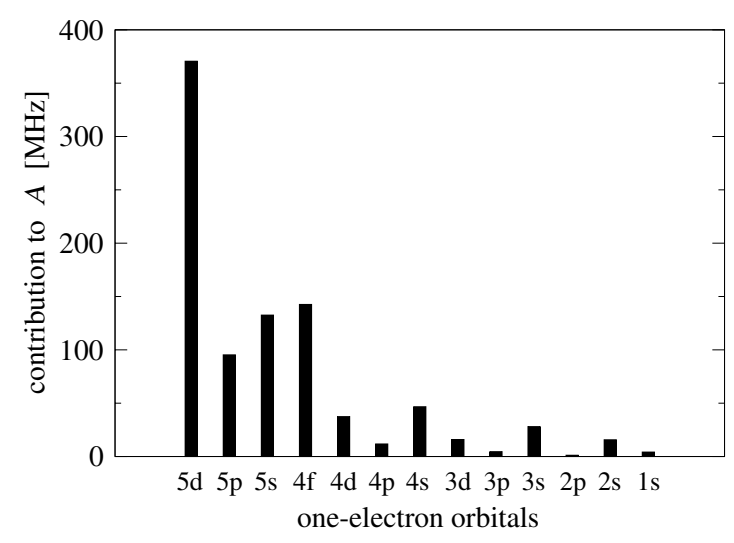

Figure 1. Contributions from sub-valence electron shells to the calculated value of $A$ of the ${ }^{2} S_{1 / 2}$ state of $A u$. The largest, direct $6 \mathrm{~s}$ term is not shown. See the caption of table 2 and section 3.2 for further details.

Table 2. Contributions from occupied orbitals to the calculated value of $A$ for the ${ }^{2} S_{1 / 2}$ state of $A u$; 'orbitals' = set of orbitals open for single and restricted double substitutions; ' $\mathrm{NCF}$ ' = size of the multiconfiguration expansion; third column $=$ value of $A$ [MHz] obtained with 'single plus restricted double substitutions' from the shells listed in the first column; last column $=$ contribution [MHz] of the first-mentioned orbital from a given set (i.e. the individual contribution of the $4 \mathrm{~d}$ orbital is in the last column of the line 4df5spd6s).

\begin{tabular}{lllr}
\hline Orbital set & NCF & $A(\mathrm{MHz})$ & Contribution \\
\hline 6s & 1 & 2344.5450 & 2344.55 \\
5d6s & 4201 & 2715.1330 & 370.59 \\
5pd6s & 7169 & 2810.4621 & 95.33 \\
5spd6s & 8269 & 2943.0173 & 132.56 \\
4f5spd6s & 12904 & 3085.6148 & 142.60 \\
4df5spd6s & 17096 & 3123.0355 & 37.42 \\
4pdf5spd6s & 20064 & 3134.7972 & 11.76 \\
4spdf5spd6s & 21164 & 3181.4858 & 46.69 \\
3d4spdf5spd6s & 25356 & 3197.4207 & 15.93 \\
3pd4spdf5spd6s & 28324 & 3202.0206 & 4.60 \\
3spd4spdf5spd6s & 29424 & 3230.0780 & 28.06 \\
2p3spd4spdf5spd6s & 32392 & 3231.2995 & 1.22 \\
2sp3spd4spdf5spd6s & 33492 & 3247.0218 & 15.72 \\
1s2sp3spd4spdf5spd6s & 34592 & 3251.2193 & 4.20 \\
\hline
\end{tabular}

total $A$ value, i.e. an order of magnitude less than the expected final accuracy in either of these two papers. Nevertheless, this contribution has been included in the $A$ value obtained within an approximation which we refer to as 'single plus restricted double substitutions' (SrD).

It should be stressed that the data in table 2 were obtained with single and restricted double substitutions, i.e. with unrestricted double and triple substitutions excluded. Therefore the contributions of the 5psd and 4spdf shells are distorted-if double and triple substitutions were included the sum of the contributions of the 5 psd and 4 spdf shells would be reduced by $25 \%$. Only the $3 \mathrm{spd}, 2 \mathrm{sp}$ and $1 \mathrm{~s}$ shells are essentially insensitive to double and triple substitutions (see section 3.3 below), therefore their contributions presented in table 2 are approximately correct. With the above caution in mind, several interesting conclusions can be drawn from table 2. All shells contribute to the final value of $A$. All contributions are positive. The orbitals with highest angular symmetry within outer shells (i.e. $5 \mathrm{~d}$ and 4 f) are very soft (i.e. easily polarizable by valence electron). Even very deep $4 \mathrm{~d}$ and $3 \mathrm{~d}$ orbitals contribute $1 \%$ and $0.5 \%$, respectively. The effect of the $5 \mathrm{~d}$ orbital is $50 \%$ larger than that of $5 \mathrm{p}$ and $5 \mathrm{~s}$ combined. The effect of the $4 \mathrm{f}$ orbital is $50 \%$ larger than that of $4 \mathrm{~d}, 4 \mathrm{p}$, and $4 s$ combined. Both the above observations indicate that orbital polarization effects [17] strongly compete with spin polarization, and within $n=5$ and $n=4$ shells orbital polarization effects dominate. Even the effect of the $3 \mathrm{~s}$ orbital is only twice as large as that of the $3 \mathrm{~d}$ orbital. Only at the very bottom (1s, $2 \mathrm{sp}, 3 \mathrm{spd}$ shells) do the spin effects dominate, although, as can be seen in figure 1 , they are an order of magnitude smaller than contributions of the outer orbitals.

\subsection{Unrestricted single and double substitutions}

Table 3 presents the effects of unrestricted double substitutions on the calculated hyperfine value $A$. They were evaluated for several different virtual sets, populated by substitutions from several occupied shells. The calculations were carried out in the configuration-interaction approach, i.e. with fixed radial functions. All orbitals and configurations described in sections 3.1 and 3.2 were included. These configurations were augmented by CSFs obtained from unrestricted double substitutions from selected occupied orbitals to a selected virtual set. The selections are described in the first three columns of table 3, as follows. The symbol in the first column is a shorthand notation. As an example, the symbol 5SD1 in the first column of the first line indicates that $\mathbf{5}$ spd6s occupied shells were opened for Single and Double substitutions to $\mathbf{1}$ virtual layer (as explained in the following two columns in table 3).

This approximation converges at the level '4SD4', i.e. with single and double substitutions from 4 spdf5spd6s shells to four layers of virtual orbitals. A comparison of lines '3SD3' with '4SD3', '3SD2' with '4SD2', and '3SD1' with '4SD1' in table 3 indicates that opening 3 spd shells for double substitutions changes the expectation value by a factor of 0.0003 , much smaller than the effect of the omitted double substitutions to the fifth and next virtual layers and still smaller than the accuracy expected in the present paper.

The lines '4SD4h1' and '4SD5h1' in table 3 denote two calculations intended to evaluate the effect of double substitutions on the fifth virtual layer. In order to save computer time they were performed with only one orbital of $h$ symmetry. The comparison indicates that the effect of double substitutions to the fifth virtual layer is about $0.5 \%$ and the effects of the subsequent shells are expected to be much smaller. Although the cumulative effect of all omitted virtual shells may be somewhat larger, we were unable to evaluate it, due to hardware and software limitations. Therefore in the present calculations the '4SD4' approximation (see table 3) was assumed as the final model involving unrestricted double substitutions. It is based on eight layers of virtual orbitals and all configurations described in sections 3.1 and 3.2 plus all configurations generated by unrestricted double substitutions from 4 spdf5spd6s occupied shells to four lowest layers of virtual orbitals. 
Table 3. Calculated value of $A$ for ${ }^{2} S_{1 / 2}$ state of $\mathrm{Au}$ as a function of multiconfiguration expansion with single and unrestricted double substitutions. All previous single and restricted double $(\mathrm{SrD})$ substitutions are included. The symbols in the first column indicate the correlation level (i.e. '5SD1' indicates single and double substitutions from 5spd6s occupied orbitals to 1 virtual orbital layer). 'NCF' $=$ size of multiconfiguration expansion; ' $1 \mathrm{v}$ ' $=1$ spdfgh; ' $2 \mathrm{v}$ ' $=2$ spdfgh; ' $3 \mathrm{v}$ ' $=3$ spdfgh.

\begin{tabular}{|c|c|c|c|c|}
\hline \multirow[b]{2}{*}{ Symbol } & \multirow[b]{2}{*}{ From } & \multicolumn{2}{|c|}{ Experiment } & \multirow{2}{*}{$\begin{array}{l}3049.660092(7) \\
A(\mathrm{MHz})\end{array}$} \\
\hline & & To & $\mathrm{NCF}$ & \\
\hline $\mathrm{SrD}$ & 1s2sp3spd4spdf5spd6s & $8 v$ & 34592 & 3251.22 \\
\hline 5SD1 & $5 s p d 6 s$ & $1 \mathrm{v}$ & 36867 & 2887.72 \\
\hline $5 \mathrm{SD} 2$ & 5 spd6s & $2 v$ & 43594 & 2754.27 \\
\hline $5 \mathrm{SD} 3$ & 5 spd6s & $3 \mathrm{v}$ & 54773 & 2729.11 \\
\hline 4SD1 & 4 spdf5spd6s & $1 \mathrm{v}$ & 50603 & 2871.01 \\
\hline 4SD2 & 4 spdf5spd6s & $2 v$ & 98002 & 2634.12 \\
\hline 4SD3 & 4 spdf5spd6s & $3 v$ & 176789 & 2581.47 \\
\hline 4SD4 & 4 spdf5spd6s & 4 spdfg $3 h$ & 265571 & 2554.80 \\
\hline 4SD4h1 & 4 spdf5spd6s & 4spdfg1h & 227579 & 2565.37 \\
\hline 4SD5h1 & 4 spdf5spd6s & 5 spdfg $1 \mathrm{~h}$ & 330379 & 2553.50 \\
\hline 4SD5 & 4 spdf5spd6s & 5 spdfg $3 h$ & 368371 & 2542.93 \\
\hline 3SD1 & 3 spd4spdf5spd6s & $1 \mathrm{v}$ & 65278 & 2871.22 \\
\hline 3SD2 & 3 spd4spdf5spd6s & $2 \mathrm{v}$ & 156102 & 2634.25 \\
\hline 3SD3 & 3 spd4spdf5spd6s & $3 v$ & 307064 & 2580.61 \\
\hline
\end{tabular}

Table 4. Various combinations of single, double and triple substitutions, which served as preliminary calibration for final series of the configuration-interaction calculations. All previous single and restricted double (SrD) substitutions were included. The symbols in the first column indicate approximations employed for double substitutions (i.e. '3SD1' indicates substitutions from 3spd4spdf5spd6s occupied shells to 1 virtual orbital layer). The symbols in the second column indicate approximations employed for triple substitutions. They are explained in third and fourth columns (i.e. 'sSDT1s' indicates triple substitutions from 4s5s6s shells to 1 virtual orbital of $s$ symmetry). 'NCF' = size of multiconfiguration expansion.

\begin{tabular}{llllll}
\hline & \multicolumn{2}{c}{ Substitutions } & \multicolumn{2}{c}{ Experiment } & $3049.660092(7)$ \\
\hline Double & & Triple & To & NCF & $A(\mathrm{MHz})$ \\
& Label & From & To & & \\
\hline SrD & sSDT1s & 4 s5s6s & 1s & 31380 & 3228.76 \\
3SD1 & sSDT1s & 4s5s6s & 1s & 67226 & 2871.19 \\
3SD1 & 5SDT1d & 5spd6s & 1spd & 76308 & 2928.33 \\
3SD1 & 4f5SDT1f & 4f5spd6s & 1spdf & 215894 & 3033.64 \\
3SD1 & 5SDT2d & 5spd6s & 2spd & 155196 & 2883.39 \\
3SD1 & spSDT3p & 4sp5sp6s & 3sp & 154519 & 2859.88 \\
\hline
\end{tabular}

Table 5. Calculated value of $A$ for the ${ }^{2} S_{1 / 2}$ state of $A u$ as a function of the size of the single, double and triple substitutions combined together. All previous single and restricted double (SrD) substitutions are included. The symbol in the first column indicates correlation level (i.e. '5SDT2fd' indicates single, double, and triple substitutions from occupied 5 spd6s shells to two virtual layers; 'fd' indicates that two virtual layers are limited to spdf and spd symmetries, respectively); 'NCF' = size of multiconfiguration expansion.

\begin{tabular}{|c|c|c|c|c|}
\hline \multirow[b]{2}{*}{ Symbol } & \multirow[b]{2}{*}{ From } & \multicolumn{2}{|c|}{ Experiment } & \multirow{2}{*}{$\frac{3049.660092(7)}{A(\mathrm{MHz})}$} \\
\hline & & To & $\mathrm{NCF}$ & \\
\hline 5SDT1d & 5 spd6s & $1 \mathrm{spd}$ & 47897 & 2951.35 \\
\hline 5SDT1f & 5 spd6s & $1 \mathrm{spdf}$ & 76016 & 3056.91 \\
\hline $5 \mathrm{SDT} 2 \mathrm{fd}$ & 5 spd6s & $2 \mathrm{spd} 1 \mathrm{f}$ & 218627 & 2866.07 \\
\hline
\end{tabular}

\subsection{Unrestricted single, double and triple substitutions}

Table 4 presents the effects of several different combinations of single, double and triple substitutions on the calculated value of $A$. The symbol in the first column denotes the configurations generated with double substitutions, as explained in section 3.3. The symbol in the second column denotes the configurations generated with triple substitutions, and it is explained in the third and fourth columns. The results presented in table 4 were used as test cases, before more elaborate, systematic and balanced calculations were subsequently carried out. These are presented in table 5 which shows the effects of unrestricted single, double and triple substitutions on the calculated hyperfine value of $A$. Similar to the procedures described in the previous section, these calculations were also carried out in the configurationinteraction approach, i.e. with fixed radial functions. All orbitals and configurations described in sections 3.1 and 3.2 were included. These were augmented by unrestricted double and triple substitutions from selected occupied orbitals to a selected virtual set. The selections are described in the first three columns of table 5 , in exactly the same manner as in section 3.3. As an example, the symbol 5SDT2fd in the first column of the third line indicates the unrestricted Single and Double and Triple substitutions from $\mathbf{5 s p d 6}$ s shells to $\mathbf{2}$ virtual layers (one virtual layer with spdf and one with spd symmetry). Because of memory requirements we were unable to converge the calculations with larger expansions than '5SDT2fd'. The density of the Hamiltonian matrix is higher in the SDT approximation than in the corresponding SD approximation of 


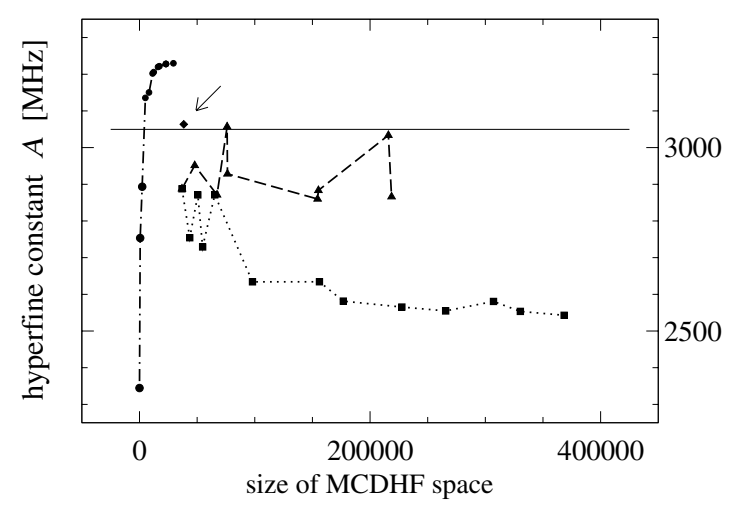

Figure 2. The magnetic dipole hyperfine constant $A$ of the $5 d^{10} 6 \mathrm{~s}$ ${ }^{2} \mathrm{~S}_{1 / 2}$ state of gold as a function of the size of multiconfiguration expansion (NCF) in three different approximations: curve with circles - single and restricted double substitutions; curve with squares - single and unrestricted double substitutions; curve with triangles - single and unrestricted double and unrestricted triple substitutions; the arrow points to the value from [8] (see the text for details); solid horizontal line-experimental value from [3].

the same size. As demonstrated in section 3.3, the calculations performed in one- and two-electron approximation converged at the '4SD4' level, i.e. with single and double substitutions from 4 spdf5spd6s orbitals to four virtual layers (compare lines '4SD3', '3SD3' and '4SD4' in table 3). Therefore it may be expected that a fully converged SDT approximation would also converge at or close to the '4SDT4' level, i.e. with single, double, and triple substitutions from 4 spdf5spd6s orbitals to four virtual layers. Such an approximation would require 6978457 configuration state functions, which exceeds the capacity of our current computer resources by more than an order of magnitude. Therefore the present calculations were terminated at '5SDT2fd' level in the approximation involving single, double and triple substitutions.

\subsection{Corrections}

All calculations were performed with the Dirac-Coulomb Hamiltonian (equation (2)). The hyperfine integrals were calculated assuming a point-dipole source. No attempt was made to evaluate the Bohr-Weisskopf correction [34] for the finite distribution of nuclear currents [35]. The small corrections arising from the Breit interaction and from the leading QED effects, together with the Bohr-Weisskopf correction, can be estimated in a similar way as in the paper by Song et al [8,36]. An inspection of their table 5 indicates that the combined effect of these three corrections is about $0.4 \%$. This is at least an order of magnitude smaller than the neglected electron correlation effects arising from two-, three- and possibly four-electron substitutions. Therefore in the present paper we neglected all three above-mentioned corrections.

\section{Results}

Figure 2 presents the dependence of the calculated value of $A$ on the size of the multiconfiguration expansion in three different approximations compared to the experimental value of Dahmen and Penselin [3]. The arrow points to the value obtained by Song et al [8]. They generated four layers of virtual orbitals in an approximation similar to ours (as in section 3.1), and appended their multiconfiguration expansion with double substitutions from $3 \mathrm{~d} 4$ spdf5spd6s orbitals to one layer of virtual orbitals (see page 478 in [8]). The curve with circles was obtained during the initial phase of our calculations, along with the generation of the virtual orbital set, in the approximation involving single and restricted double substitutions (see sections 3.1 and 3.2). An inspection of table 1 reveals that this approximation has converged to within $0.1 \%$. The curve with squares represents the data from table 3, i.e. in the approximation involving single and unrestricted double substitutions. This is also an essentially converged calculation, as discussed in section 3.3. The curve with triangles represents the data obtained with single and unrestricted double and unrestricted triple substitutions (see section 3.4). There is a point at the horizontal axis, around 200000 configuration state functions, where the SD curve becomes essentially flat, which means that the double substitutions converged at this stage. Therefore the effects which induce the oscillations of the SDT curve with larger multiconfiguration expansions are expected to come mainly from the triple substitutions through their indirect effect on the double and single substitutions. If there are nonnegligible three-particle effects, then the oscillations of the SDT curve are expected to extend further than oscillations of the SD curve for the following reasons. Each triangle on the SDT curve represents a CI calculation in which one or more occupied shells were opened for single, double and triple substitutions. Therefore the number of configuration state functions increases rapidly, when going from point to point (or rather, from triangle to triangle) on the SDT curve. This increase is often an order of magnitude larger than in the corresponding CI calculation involving only single and double substitutions. Therefore the SDT curve could not be extended as far as the SD curve. The overall convergence is slow compared to other heavy atoms. Such slow convergence is a common property of atoms with open valence shells of s symmetry (see, e.g., the papers $[28,29]$ where up to 18 orbital layers were necessary to converge the values of hyperfine constants of lithium and lithium-like beryllium). The convergence of double and triple substitutions in the present calculations is additionally slowed down by the fact that virtual orbitals are optimized to accommodate the dominant single-electron contributions (plus restricted double substitutions) to the hyperfine expectation value (see sections 1 and 3.1), and they are not necessarily optimal for unrestricted double and triple substitutions.

\section{Conclusions}

We have shown that much more complicated electron correlation terms than those included by Song et al [8] are required for treating the magnetic dipole hyperfine coupling constant $A$ for the ground state of the Au atom. As explained in section 3.4 , we were unable to fully converge the calculations 
with triple substitutions. Nevertheless, the results obtained in the present paper constitute clear evidence that two- and threeelectron correlation effects at the ground state of the gold atom are important and cannot be neglected. Therefore, double and triple substitutions must be taken into account in accurate $a b$ initio calculations of the magnetic dipole hyperfine structure constant of the ground state of atomic gold. All occupied shells contribute to the final value of $A$. Unanswered remains the question, how large is the contribution of quadruple substitutions, which may be expected to indirectly affect the contribution of double substitutions.

\section{Acknowledgments}

This work was supported by the Polish Ministry of Science and Higher Education (MNiSW) in the framework of scientific grant No 1 P03B 11030 awarded for the years 2006-2009. PJ acknowledges support from the Swedish Research Council (Vetenskapsrådet). PP belongs to the Finnish Center of Excellence in Computational Molecular Science (CMS). The visits of JB at Helsinki were supported by The Academy of Finland. The large scale calculations were performed on the Linux cluster at the National Institute of Standards and Technology (NIST), USA. JB would like to express his gratitude for the hospitality which was extended to him during his visits to the Chemistry Department of the University of Helsinki and the Atomic Spectroscopy Group at NIST.

\section{References}

[1] Pyykkö P and Desclaux J P 1979 Acc. Chem. Res. 12276

[2] Autschbach J, Siekierski S, Seth M, Schwerdtfeger P and Schwarz W H E 2002 J. Comp. Chem. 23804

[3] Dahmen H and Penselin S 1967 Z. Phys. 200456

[4] Pyykkö P 2004 Angew. Chem. Int. Ed. 434412 Pyykkö P 2004 Angew. Chem. 1164512 (edition in German)

[5] Pyykkö P 2005 Inorg. Chim. Acta 3584113

[6] Pyykkö P 2008 Chem. Soc. Rev. (at press)

[7] Yakobi H, Eliav E and Kaldor U 2007 J. Chem. Phys. 126184305

[8] Song S Q, Wang G F, Ye A P and Jiang G 2007 J. Phys. B: At. Mol. Opt. Phys. $\mathbf{4 0} 475$
[9] Lindgren I 1984 Rep. Prog. Phys. 47345

[10] Wybourne B G 1965 Spectroscopic Properties of Rare Earths (New York: Wiley) p 148

[11] Bieroń J 2005 J. Phys. B: At. Mol. Opt. Phys. 382221

[12] Bieroń J, Pyykkö P and Jönsson P 2005 Phys. Rev. A 71012502

[13] Bieroń J, Froese Fischer C and Godefroid M 2002 J. Phys. B: At. Mol. Opt. Phys. 353337

[14] Bieroń J and Pyykkö P 2001 Phys. Rev. Lett. 87133003

[15] Pyykkö P 1988 Chem. Rev. 88563

[16] Panigrahy S N, Dougherty R W, Das T P and Andriessen J 1991 Phys. Rev. A 44121

[17] Froese Fischer C, Brage T and Jönsson P 1997 Computational Atomic Structure. An MCHF Approach (Bristol: Institute of Physics Publishing) p 171

[18] Godefroid M, Meulebeke G V, Jönsson P and Froese Fischer C 1997 Z. Phys. D 42193

[19] Engels B 1993 Theor. Chim. Acta 86429

[20] Jönsson P, Ynnerman A, Froese Fischer C, Godefroid M R and Olsen J 1996 Phys. Rev. A 534021

[21] Malkin I, Malkina O L, Malkin V G and Kaupp M 2004 Chem. Phys. Lett. 396268

[22] Malkin E, Malkin I, Malkina O L, Malkin V G and Kaupp M 2006 Phys. Chem. Chem. Phys. 84079

[23] Itano W M 2006 Phys. Rev. A 73022510

[24] Jönsson P, He X, Froese Fischer C and Grant I P 2007 Comput. Phys. Commun. 177597

[25] Grant I P 2007 Relativistic Quantum Theory of Atoms and Molecules: Theory and Computation (New York: Springer)

[26] Grant I P 1994 Comput. Phys. Commun. 8459

[27] Lindgren I and Rosén A 1974 Case Stud. At. Phys. 4150

[28] Bieroń J, Jönsson P and Froese Fischer C 1996 Phys. Rev. A 532181

[29] Bieroń J, Jönsson P and Froese Fischer C 1999 Phys. Rev. A 603547

[30] Grant I P 1988 Relativistic Atomic Structure Calculations (Methods in Computational Chemistry vol 2) ed S Wilson (New York: Plenum)

[31] Dyall K G, Grant I P, Johnson C T, Parpia F A and Plummer E P 1989 Comput. Phys. Commun. 55425

[32] Raghavan P 1989 At. Data Nucl. Data Tables 42189

[33] Dougherty R W, Panigrahy S N, Das T P and Andriessen J 1993 Phys. Rev. A 472710

[34] Bohr A and Weisskopf F 1950 Phys. Rev. 7794

[35] Jönsson P, Parpia F A and Froese Fischer C 1996 Comput. Phys. Commun. 96301

[36] Kopfermann H 1958 Nuclear Moments (New York: Academic) 